Published in "Experimental Gerontology 73: 78-85, 2016"

which should be cited to refer to this work.

\title{
Postural challenge affects motor cortical activity in young and old adults
}

\author{
Selma Papegaaij a,*, Wolfgang Taube ${ }^{\mathrm{b}}$, Helco G. van Keeken ${ }^{\mathrm{a}}$, Egbert Otten ${ }^{\mathrm{a}}$, \\ Stéphane Baudry ${ }^{\text {c }}$, Tibor Hortobágyi a,d \\ ${ }^{a}$ University of Groningen, University Medical Center Groningen, Center for Human Movement Sciences, Antonius Deusinglaan 1, 9713AV, Groningen, The Netherlands \\ ${ }^{\mathrm{b}}$ University of Fribourg, Department of Medicine, Ch. du Musée 8, CH-1700, Fribourg, Switzerland \\ c Université Libre de Bruxelles, Faculty for Motor Sciences, Laboratory of Applied Biology, CP 640, Route de Lennik 808, 1070 Brussels, Belgium \\ ${ }^{\mathrm{d}}$ Department of Sport, Exercise and Rehabilitation, Northumbria University, Newcastle-upon-Tyne NE1 8ST, United Kingdom
}

\begin{abstract}
When humans voluntarily activate a muscle, intracortical inhibition decreases. Such a decrease also occurs in the presence of a postural challenge and more so with increasing age. Here, we examined age-related changes in motor cortical activity during postural and non-postural contractions with varying levels of postural challenge. Fourteen young (age 22) and twelve old adults (age 70) performed three conditions: (1) voluntary contraction of the soleus muscle in sitting and (2) leaning forward while standing with and (3) without being supported. Subthreshold transcranial magnetic stimulation was applied to the soleus motor area suppressing ongoing EMG, as an index of motor cortical activity. The area of EMG suppression was $~ 60 \%$ smaller $(p<0.05$ ) in unsupported vs. supported leaning and sitting, with no difference between these latter two conditions ( $p>0.05$ ). Even though in absolute terms young compared with old adults leaned farther $(p=0.018)$, there was no age effect or an age by condition interaction in EMG suppression. Leaning closer to the maximum without support correlated with less EMG suppression ( $r$ o $=-0.44, \mathrm{p}=0.034$ ). We conclude that the critical factor in modulating motor cortical activity was postural challenge and not contraction aim or posture. Age did not affect the motor control strategy as quantified by the modulation of motor cortical activity, but the modulation appeared at a lower task difficulty with increasing age.
\end{abstract}

\section{Introduction}

Although historical studies in intact and decerebrate animals identified subcortical neural circuits, especially spinal reflexes, as centers to control upright standing (Sherrington, 1910; Magnus, 1926), recent studies have provided evidence for the involvement of the motor cortex (Tokuno et al., 2009; Taube et al., 2006; Horak et al., 1989; Malouin et al., 2003). However, it remains elusive if the motor cortical control differs between voluntary and postural contractions and if age affects this control (Papegaaij et al., 2014a).

When humans voluntarily activate a muscle, the magnitude of short-interval intracortical inhibition (SICI) decreases (Ridding et al., 1995; Rantalainen et al., 2013; Sharples and Kalmar, 2012; Ortu et al., 2008). It is thought that inhibitory intracortical circuits modulate the excitability of the cortical neurons that project to the spinal motor neurons of the muscles involved in the task (Reis et al., 2008). The degree of reduction in intracortical inhibition is related to contraction intensity (Rantalainen et al., 2013; Ortu et al., 2008), contraction type (Howatson et al., 2011), and whether the movement starts or ends (Sidhu et al., 2013). SICI is also modulated during postural contractions,

\footnotetext{
* Corresponding author.

E-mail address: s.papegaaij@umcg.nl (S. Papegaaij).
}

defined as contractions with the aim to maintain a certain posture, as shown by a reduction in SICI in the soleus muscle during standing as compared with sitting (Soto et al., 2006).

In addition to the aim of the contraction (postural vs. non-postural), postural challenge may also affect inhibition. We define postural challenge as the degree of difficulty one encounters in holding a specific body position. SICI in the tibialis anterior is lower during standing as compared with sitting, even though this muscle is only weakly activated during these tasks (Obata et al., 2014). Such a context-related reduction in SICI suggests that increased postural challenge is coupled with higher motor cortical excitability. A limitation of comparing sitting with standing is that not only postural challenge but also posture itself is different between conditions, which may affect motor cortical excitability (Ginanneschi et al., 2005; Dominici et al., 2005). However, also when normal standing was compared with supported standing, motor cortical excitability in the soleus muscle was higher (Tokuno et al., 2009) and SICI was lower (Papegaaij et al., submitted for publication) during normal standing. The emerging picture is that the motor cortex is involved in postural contractions to control upright standing and that its excitability increases with increasing postural challenge.

There is some evidence that the postural challenge-related increase in motor cortical excitability increases with age. When healthy adults stood on a rigid surface and then on foam, this increase in postural 
challenge resulted in a decrease in SICI in old but not in young adults' tibialis anterior muscle (Papegaaij et al., 2014b). However, this age by condition interaction was not present in normal standing, a relatively easy postural task (Papegaaij et al., submitted for publication; Baudry et al., 2015). Therefore, it is unclear if the modulation between a stable and unstable condition in old adults reflects a different motor control strategy or different relative task difficulty (i.e., the same task being more difficult for old than young adults). Moreover, it is unclear if age affects the motor cortical control of postural and non-postural contractions.

Therefore, the aim of the current study was to examine age-related changes in motor cortical activity during non-postural and postural contractions in a postural challenging and non-challenging context. Subjects were asked to (1) voluntarily contract the soleus muscle during sitting (SIT), (2) lean forward during standing, with support at the chest (SL), and (3) lean forward during standing, without support (UL). These conditions allowed us to disentangle the effects of contraction aim, postural challenge, and posture, and to investigate the interaction with age. To examine motor cortical activity, we applied transcranial magnetic stimulation pulses at subthreshold intensities (subTMS). Such pulses suppress ongoing electromyographic (EMG) activity through the activation of inhibitory intracortical circuits (Davey et al., 1994).

Based on fMRI and TMS studies, suggesting that motor control relies more on cortical structures in old compared with young adults (Papegaaij et al., 2014b; Mattay et al., 2002; Heuninckx et al., 2008; Baudry et al., 2014), we hypothesized an age by condition interaction in motor cortical activity as indexed by TMS-induced EMG suppression. We also expected that the pattern of changes in EMG suppression between the conditions would provide insights into which of the three factors (contraction aim, postural challenge, posture) is critical in modulating motor cortical activity (see Fig. 1). If contraction aim is a critical factor, we would expect a gradual change in TMS-induced EMG suppression from sitting to supported leaning to unsupported leaning. We note that during supported leaning the contraction was a combined postural and non-postural contraction. As support was provided only at the chest, a postural contraction was still needed to prevent the body from buckling at the hip. To reach the target EMG level subjects were instructed to add a small amount of voluntary activation to the ongoing activation produced by the postural contraction, resulting in a mix of voluntary and postural soleus activation. If postural challenge is a critical factor in modulating motor cortical activity, we would expect similar TMS-induced EMG suppression in sitting and supported leaning, with different suppression in unsupported leaning. If posture is critical, we would expect supported and unsupported leaning to be similar, with different TMS-induced EMG suppression in sitting.

\section{Materials and methods}

\subsection{Subjects}

Sixteen young (20-31 years) and seventeen old adults (64-83 years) participated in the study (Table 1). Participants were free of neurological or orthopedic conditions, non-dental associated metal within the cranium, did not take neuroactive drugs or drugs known to affect balance, and reported to be not pregnant. General cognitive function was assessed by the Mini-Mental State Examination (MMSE) and physical activity level by the Short Questionnaire to Assess Health-enhancing physical activity (SQUASH). Lower extremity function was evaluated by the Short Physical Performance Battery (SPPB), including standing balance, walking speed and chair stand tests (Guralnik et al., 1994). Before the experiment, all subjects signed an informed consent document approved by the Medical Ethics Committee of the University Medical Center Groningen.

\subsection{Experimental protocol}

The experiments were conducted in one, 3-hour-long session. Subjects were standing on two force platforms with the feet in a selfselected position that was marked on the force platforms to ensure consistent positioning throughout the experiment (intermalleolar distance, young: $17.3 \pm 1.1 \mathrm{~cm}$, old: $14.0 \pm 1.1 \mathrm{~cm}$ ). SubTMS was applied while subjects subsequently performed the following three tasks in a randomized order: unsupported leaning (UL), supported leaning (SL), and sitting (SIT). During UL, subjects were instructed to lean forward by dorsal flexing their ankles while keeping the rest of the body straight. Subjects received online feedback by watching a red ball moving over a black background, representing the movements of the CoP in the anterior-posterior direction. Upward movement of the ball corresponded to a forward shift of the CoP. A dark green horizontal line was set as a CoP target at $75 \%$ of the maximum voluntary and unaided forward lean. Thus, task difficulty was adjusted to individual skill level. Two light green horizontal lines at $70 \%$ and $80 \%$ of the maximum cued the subjects to keep the CoP on the dark green target line and within the

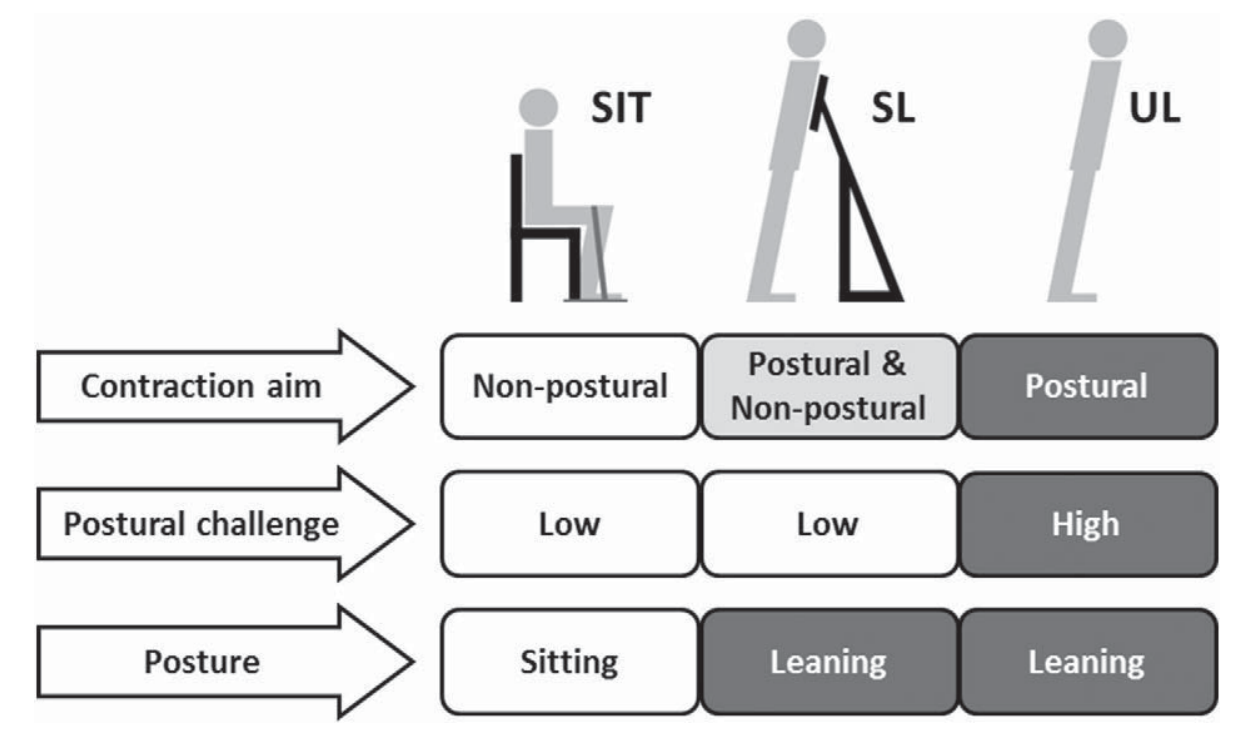

Fig. 1. A summary of experimental conditions and conceptual interactions between three different conditions (sitting - SIT, supported leaning - SL, unsupported leaning - UL) that could influence intracortical inhibition (contraction aim, postural challenge, posture). 
Table 1

Subject characteristics.

\begin{tabular}{lll}
\hline & Young adults & Old adults \\
\hline Age (years) & $22.1 \pm 0.9$ & $71.3 \pm 1.1$ \\
Sex $($ male; female) & $8 ; 8$ & $7 ; 10$ \\
Height $(\mathrm{m})^{*}$ & $1.78 \pm 0.02$ & $1.72 \pm 0.02$ \\
BMI $\left(\mathrm{kg} / \mathrm{m}^{2}\right)^{*}$ & $22.3 \pm 0.4$ & $25.3 \pm 0.8$ \\
SPPB score & $12.0 \pm 0.0$ & $11.5 \pm 0.2$ \\
MMSE score* & $29.8 \pm 0.1$ & $28.4 \pm 0.3$ \\
SQUASH: & & \\
Total score & $8904 \pm 798$ & $7636 \pm 630$ \\
Light $(\mathrm{min} / \mathrm{w})^{*}$ & $1859 \pm 180$ & $851 \pm 135$ \\
$\quad$ Moderate $(\mathrm{min} / \mathrm{w})$ & $321 \pm 72$ & $199 \pm 41$ \\
Heavy $(\mathrm{min} / \mathrm{w})^{*}$ & $243 \pm 44$ & $516 \pm 71$ \\
\hline
\end{tabular}

Values are mean \pm SE, unless denoted differently. BMI: body mass index, SPBB: short physical performance battery (max. score of 12), MMSE: mini mental state examination (max. score of 30), SQUASH: short questionnaire to assess health-enhancing physical activity. Total score is minutes per week $\times$ intensity of the activity. The amount of light, moderate and heavy exercise is expressed in minutes per week.

* $\mathrm{p}<0.05$ : denotes a significant difference between young and old adults.

$5 \%$ band around it. Subjects were instructed to try to keep the ball on the target line. During SL, the body position was kept the same as during UL but the subjects were supported at chest level by a sturdy and adjustable custom-made frame. Subjects were instructed to rest against the frame with full body weight. During SL and SIT, subjects matched the soleus EMG activity with that recorded during UL. In these conditions, instead of the red ball, subjects received online feedback in the form of the rectified and smoothed EMG signal displayed together with a horizontal target line. Subjects were instructed to try to keep the line of the ongoing EMG on the target line. In SL, subjects matched the EMG recorded in UL by putting more pressure on the right forefoot without changing posture. In SIT, the EMG matching was accomplished by raising the heel against resistance from a strap fixed tightly around the forefoot and over the knee. After each condition, subjects were asked to fill in the Borg Rating of Perceived Exertion (RPE) scale (ranging from 6 to 20) (Borg, 1970). At the beginning of the experiment subjects were instructed to stand quietly for $10 \mathrm{~s}$. These data were used as a reference to quantify forward lean and determine differences in joint angles between standing and leaning. Before and after the experiment, subjects performed another $30 \mathrm{~s}$ of UL without stimulation to determine if the experimental protocol caused fatigue.

\subsection{Data acquisition}

Parallel-bar EMG sensors (TrignoTM Wireless System, Delsys, Natick, MA, USA) were placed on the muscle belly of the soleus muscle of the preferred leg. The preference leg was determined by testing which foot was used when kicking a football, pushing an object with the foot, and stamping on the ground (Hebbal and Mysorekar, 2006). To minimize impedance, the skin was shaved, abraded with fine-grain sandpaper, and cleaned with alcohol. Surface EMG was pre-amplified $300 \times$ in the sensor and then further amplified by a factor of 3.03 in the base station, resulting in a total signal amplification of 909. Signals were sampled at $4 \mathrm{kHz}$ and bandpass filtered with a second order Butterworth filter $(10-1000 \mathrm{~Hz})$ using data acquisition interface and software (Power 1401 and Spike 7, Cambridge Electronics Design, Cambridge, UK). While seated, subjects performed two maximal voluntary contractions (MVC) of the soleus, with resistance from the same strap used during the sitting condition. The maximal EMG during the MVC was identified using a root-mean-square (RMS) algorithm with a 50-ms moving window.

An 11-video-camera motion analysis system (Vicon, Oxford, UK) recorded spatial coordinates of reflective markers placed on the right acromion, greater trochanter, lateral femoral epicondyle, lateral malleolus and fifth metatarsophalangeal joint. Two force plates (Bertec 4060-08, Columbus, OH, USA) were used to measure CoP displacement.
Kinematic and CoP data was sampled at $100 \mathrm{~Hz}$ and zero-phase filtered using a fourth order low-pass Butterworth filter with a cut off frequency of $10 \mathrm{~Hz}$.

\subsection{Transcranial magnetic stimulation}

A double cone coil (inner loop diameter $110 \mathrm{~mm}$ ) connected to a Magstim $200^{2}$ and Bistim2 (Magstim, Whitland, UK) was used to magnetically stimulate the primary motor cortex contralateral to the preferred leg. The coil, held by the experimenter, was positioned so that the current in the coil flowed in an anterior-to-posterior direction. The hotspot for the soleus muscle was located by moving the coil in a systematic mannerlaterally and posteriorly from the vertex in steps of $0.5 \mathrm{~cm}$. This hotspot was marked on the scalp to enable the experimenter to hold the coil in the same position throughout the experiment. Active motor threshold (aMT) was defined as the minimum intensity at which an MEP above bEMG could be evoked in three out of five consecutive trials and was determined during SIT with subjects matching the soleus EMG activity recorded during UL. The MEP's recorded during this procedure were used to determine MEP onset. Stimulation intensity was then decreased to $80 \%$ of aMT and single pulses were given every $1.5 \mathrm{~s}$. Responses were rectified and averaged over 20-40 trials, depending on the stability of the signal. Even though stimulation intensity was subthreshold, when averaging over multiple trials a facilitation in the EMG signal can sometimes be observed. Stimulation intensity was adjusted to get the greatest amount of TMS-induced EMG suppression without preceding facilitation. Subjects then received 4-6 blocks of 20 pulses per condition using this stimulation intensity. Rest periods of $30 \mathrm{~s}$ were given between blocks and rest periods of 5 min between conditions. As in previous studies using subTMS (Davey et al., 1994; Petersen et al., 2001; Zuur et al., 2010), two young and five old adults were excluded because they did not exhibit suppression without a preceding facilitation in all conditions.

\subsection{Data analysis}

Custom made scripts (Mathworks Matlab R2009b) were used for data analysis. EMG traces before and after the TMS pulses were rectified and averaged across trials. BEMG was calculated by averaging the rectified EMG from the $300 \mathrm{~ms}$ time window before stimulation. For every participant the MEP onset was determined from the data with suprathreshold TMS pulses. The onset of suppression after subTMS was defined as the first time point where the averaged EMG response was below the level of bEMG for at least $5 \mathrm{~ms}$ in the $30 \mathrm{~ms}$ time window starting at the MEP onset. The end of suppression was defined as the first time point where the averaged EMG response was above bEMG for longer than $1 \mathrm{~ms}$. In two young and two old subjects this algorithm did not find any EMG suppression during UL, although it did find EMG suppression during SIT and SL. In these cases we determined the onset and end of suppression manually by visual inspection. Exclusion of these subjects did not affect the main results. We quantified EMG suppression as a product of the mean amplitude of suppression in percent of bEMG and the duration of suppression in ms (i.e., Amp $\mathrm{Anrm}^{*} \mathrm{~ms}$ ). In most subjects we also observed a facilitation in EMG after the suppression. Quantification of the magnitude of this facilitation was done similarly as for the suppression. Finally, to assess fatigue, median frequency (MDF) of the EMG signal during unsupported leaning at the beginning and the end of the experiment was calculated.

Leaning performance was quantified by variability (SD) in the anterior-posterior position of the CoP and error from the target (absolute difference between mean $\mathrm{CoP}$ position and the target). The percentage lean was calculated by expressing the average of CoP position during leaning as a percentage of the maximum lean. Lastly, leaning strategy was quantified by the difference in ankle, knee and hip joint angles between leaning and standing, derived from reflective marker position data. 


\subsection{Statistical analysis}

All variables were checked for normal distribution prior to analysis. Male/female proportions were compared between young and old adults using the chi-square test. Other subject characteristics (age, height, BMI, SPPB score, MMSE score, SQUASH scores) and behavioral measures during unsupported leaning (CoP variability, CoP error, maximum lean, percentage lean, joint angles) were compared between young and old adults using independent t-tests. An Age (young, old) by Condition (sitting, supported leaning, unsupported leaning) mixed model ANOVA was used for the analysis of bEMG, EMG suppression and EMG facilitation. Results showing a significant condition effect were subjected to a post hoc Tukey's test. In case of violation of the assumption of sphericity, a Greenhouse-Geisser correction was applied. The same analysis was repeated for EMG suppression and facilitation with bEMG as a covariate. An Age (young, old) by Time (pre, post) mixed model ANOVA was used for the analysis of EMG MDF during leaning before and after the experiment. To calculate correlation between EMG suppression and behavior, EMG suppression during unsupported leaning was expressed as a percentage of EMG suppression during supported leaning. Because of non-normal distribution of this variable, correlations between EMG suppression and percentage lean, $\mathrm{CoP}$ variability, CoP error and bEMG were determined using the Spearman correlation coefficient.

\section{Results}

\subsection{Behavioral results}

There was no difference in percentage of lean between young and old adults $(\mathrm{t}(11.7)=0.1, \mathrm{p}=0.931)$, with young adults leaning to $74.4 \pm 0.2 \%$ and old adults to $74.4 \pm 0.8 \%$ of their maximum. The maximum lean relative to average CoP position during standing was farther in young $(92 \pm 8 \mathrm{~mm})$ than in old $(66 \pm 6 \mathrm{~mm})$ adults $(\mathrm{t}(21)=2.6, \mathrm{p}=$ $0.018)$, even after correction for height $(\mathrm{t}(21)=2.3, \mathrm{p}=0.033)$. Also, leaning performance was worse in old compared with young adults, with greater CoP variability (Young: $3.0 \pm 0.3 \mathrm{~mm}$, Old: $4.2 \pm$ $0.3 \mathrm{~mm} ; \mathrm{t}(21)=-2.7, \mathrm{p}=0.013$ ) and error from the target (Young: $0.6 \pm 0.1 \mathrm{~mm}$, Old: $1.5 \pm 0.2 \mathrm{~mm} ; \mathrm{t}(12.5)=-3.7, \mathrm{p}=0.003)$.

Despite instructions to lean from the ankle, leaning strategies were slightly different between the two groups. Old adults showed a greater difference in hip angle between upright standing and leaning than young adults $(\mathrm{t}(14)=-2.5, \mathrm{p}=0.023)$ and a trend towards a smaller difference in the ankle angle ( $t(19)=1.7, p=0.099$ ). Differences in knee angle between standing and leaning were similar between the groups $(\mathrm{t}(19)=0.3, \mathrm{p}=0.794)$.

\subsection{EMG activity}

As intended, the level of EMG activity did not change between conditions $(\mathrm{F}(1.3,30.7)=0.03, \mathrm{p}=0.907)$, but was higher in old $(21.2 \pm 2.2 \%$ of MVC) compared with young $(11.6 \pm 1.3 \%$ of MVC $)$ adults $(\mathrm{F}(1,24)=15.1, \mathrm{p}=0.001)$. There was no group by condition interaction $(\mathrm{F}(1.3,30.7)=2.3, \mathrm{p}=0.130)$.

\subsection{TMS measures}

Active motor threshold and TMS stimulation intensity were higher in old (aMT: $53 \pm 3 \%$, stimulation intensity: $37 \pm 2 \%$ ) compared with young (aMT: $45 \pm 2 \%$, stimulation intensity: $30 \pm 2 \%$ ) adults $(\mathrm{t}(24)=-2.2, \mathrm{p}=0.036 ; \mathrm{t}(24)=-2.5, \mathrm{p}=0.02)$.

Figs. 2 and 3 show respectively single subject and group data of EMG suppression and facilitation. The onset latency of EMG suppression was $42.6 \pm 1.4 \mathrm{~ms}$ in young and $48.4 \pm 2.2 \mathrm{~ms}$ in old adults, with a difference of $5.7 \pm 0.8 \mathrm{~ms}$ in young and $7.4 \pm 1.7 \mathrm{~ms}$ in old adults between EMG suppression onset and MEP onset. There was a condition effect on the area of EMG suppression $(F(2,48)=23.3, p<0.001)$. Tukey's post hoc tests revealed that the area of EMG suppression was $~ 60 \%$ smaller in UL compared with SL and SIT ( $p<0.05)$. EMG suppression was similar in SL and SIT $(p>0.05)$. There were no group effects or condition by group interaction effects on EMG suppression $(\mathrm{F}(1,24)=2.1, \mathrm{p}=$ $0.158 ; \mathrm{F}(2,48)=1.7, \mathrm{p}=0.188)$. None of these results were affected by the addition of bEMG as a covariate.

The onset latency of EMG facilitation was $57.1 \pm 1.4 \mathrm{~ms}$ in young and $67.8 \pm 3.8 \mathrm{~ms}$ in old adults, with a difference of $20.3 \pm 1.4 \mathrm{~ms}$ in young and $26.8 \pm 3.2 \mathrm{~ms}$ in old adults between EMG facilitation onset and MEP onset. EMG facilitation did not change between conditions ( $F(1.6$, $38.6)=0.03, p=0.954)$. There was a group effect on EMG facilitation $(\mathrm{F}(1,24)=6.4, \mathrm{p}=0.018)$, with $\sim 40 \%$ lower facilitation in old compared with young adults. There was no condition by group interaction on EMG facilitation $(F(1.6,38.6)=0.9, p=0.386)$. When EMG facilitation was adjusted for bEMG, there were no significant condition, group or interaction effects. In old adults, but not in young adults, EMG facilitation and suppression across all conditions were positively correlated $(\mathrm{r}=0.48, \mathrm{p}=0.003)$.

As interstimulus intervals were relatively short, we tested whether the train of TMS pulses induced changes in motor cortical activity by comparing the first and last 40 trials of each condition. No significant differences were found in EMG suppression (SIT: $\mathrm{t}(25)=-0.6, \mathrm{p}=$ 0.570 , SL: $\mathrm{t}(24)=0.4, \mathrm{p}=0.664$, UL: $\mathrm{t}(24)=-0.2, \mathrm{p}=0.881$ ) or EMG facilitation (SIT: $\mathrm{t}(25)=-0.2, \mathrm{p}=0.833$, SL: $\mathrm{t}(24)=-0.3$, $\mathrm{p}=0.779$, UL: $\mathrm{t}(24)=-0.1, \mathrm{p}=0.920)$.

\subsection{Correlations}

Although the target lean was set at $75 \%$ of the maximum, there was still some individual variety in percentage lean, ranging from 70.2 to 79.1\%. Participants leaning closer to their maximum showed less EMG suppression during UL as a percentage of EMG suppression during SL (rho $=-0.44, p=0.034$; Fig. 4), even when controlled for bEMG during UL (rho $=-0.53, \mathrm{p}=0.012$ ). There was, however, no correlation between EMG suppression and leaning performance, quantified by CoP variability ( $r$ o $=-0.12, \mathrm{p}=0.590$ ) and error from the target (rho $=-0.14, \mathrm{p}=0.526$ ).

\subsection{Fatigue}

There were no signs of fatigue during the experiment. There was no change in EMG MDF during leaning before and after the experiment $(F(1,24)=2.1, p=0.164)$ and the Borg rating of perceived exertion were low (Young: SIT: $11.0 \pm 0.6$, SL: $9.5 \pm 0.7$, UL: $9.6 \pm 0.5$; Old: SIT: $9.7 \pm 0.7$, SL: $10.0 \pm 0.7$, UL: $9.1 \pm 0.5)$.

\section{Discussion}

We examined age-related changes in motor cortical activity during postural and non-postural contractions in a postural challenging and non-challenging context. The main finding was a decrease in TMSinduced EMG suppression during UL compared with SL and SIT in both age groups. Moreover, leaning closer to the personal maximum correlated with a greater decrease in EMG suppression. The results suggest that postural challenge, and not contraction aim or posture, was the critical factor in modulating motor cortical activity. As quantified by the modulation in EMG suppression, age did not affect motor control strategies under the current experimental conditions, in which the task was adjusted to individual skill level.

\subsection{Cortical mechanisms}

The subTMS method was first described by Davey et al. (1994), and subsequent experiments using a variety of techniques supported the idea that the TMS-induced EMG suppression originates from activation of intracortical inhibitory circuits, reducing motor cortical output 

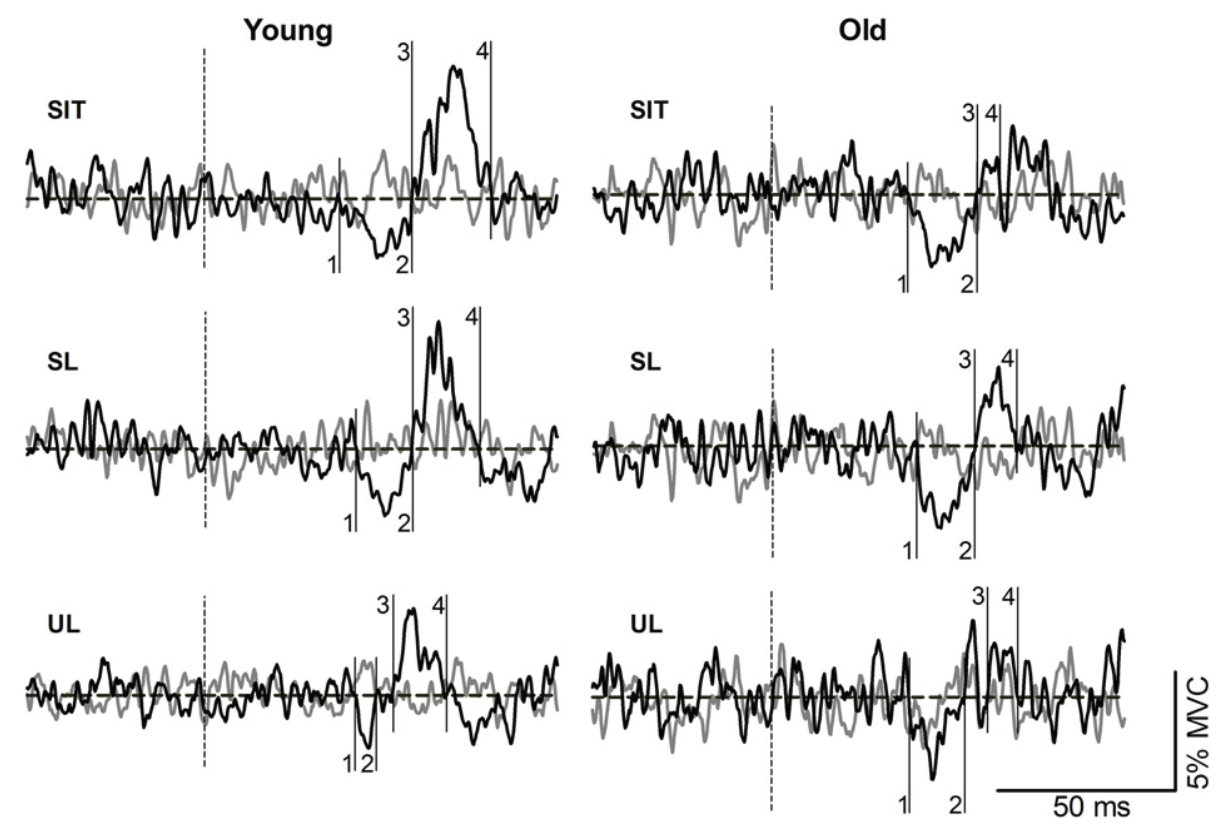

Fig. 2. Representative responses in the soleus to subthreshold transcranial magnetic stimulation (TMS) of one young ( 30 years) and one old (70 years) adult during sitting (SIT), supported leaning (SL), and unsupported leaning (UL). Waveforms represent rectified EMG traces averaged over 120 trials before the TMS pulse (gray line) and after the TMS pulse (black line). The horizontal dashed line represents the mean level of background EMG. The vertical lines represent the time of the TMS pulse (dashed line), the onset of EMG suppression (1), the end of EMG suppression (2), the onset of EMG facilitation (3), and the end of EMG facilitation (4).

(Davey et al., 1994; Petersen et al., 2001; Di Lazzaro et al., 1998; Classen and Benecke, 1995). For example, transcranial electrical stimulation, thought to activate the axons of the corticospinal neurons directly, does not suppress ongoing EMG (Petersen et al., 2001). Moreover, subTMS produces no recognizable descending volleys measured with high cervical, epidural electrodes (Di Lazzaro et al., 1998). Together these studies suggest that subTMS only activates motor cortical neurons with minimal or no influences from segmental circuits on the TMSinduced EMG suppression.

Changes in TMS-induced EMG suppression can be attributed to changes in the cortical contribution to the ongoing EMG or changes in intracortical inhibition (Seifert and Petersen, 2010). When the cortical contribution to the EMG is reduced, inhibition of the corticospinal pathway will have a smaller influence on the EMG, resulting in less TMS-induced EMG suppression. Alternatively, reduced excitability of intracortical inhibitory circuits (or increased excitability of intracortical facilitatory circuits) would also result in less TMS-induced EMG suppression. We have two reasons to favor the last explanation in the current study. First, similar changes in intracortical inhibition between postural tasks have been found using paired pulse TMS (Obata et al., 2014; Papegaaij et al., submitted for publication, 2014b). Second,

\section{A}

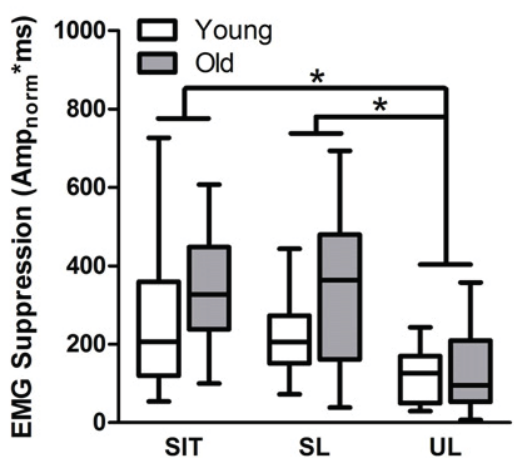

greater forward lean was associated with greater reductions in EMG suppression. It seems unlikely that one would reduce cortical contribution when getting closer to the boundaries of stability. We therefore propose that the modulation in TMS-induced EMG suppression found in the current study was related to modulation in intracortical circuits and not to changes in cortical contribution to the ongoing EMG.

\subsection{Postural challenging and non-challenging context}

Several lines of evidence suggest that the level of muscle activation may not be the only factor underlying the modulation of corticospinal excitability and intracortical inhibition. The nature of the task, i.e., how and in what context a muscle is used, also seems to contribute to this modulation. For example, in exploring the basic neural processes involved in the functional linking between motor cortical points in the ketamine-anesthetized cat, pharmacological and electrical microstimulation manipulations revealed functional connections in the recruitment of muscle synergies (Schneider et al., 2002). In healthy humans, there was a context-dependent modulation of SICI across shoulder, elbow, and finger muscles during the execution of a pointing task (Devanne et al., 2002). There was also a functional coupling

\section{B}

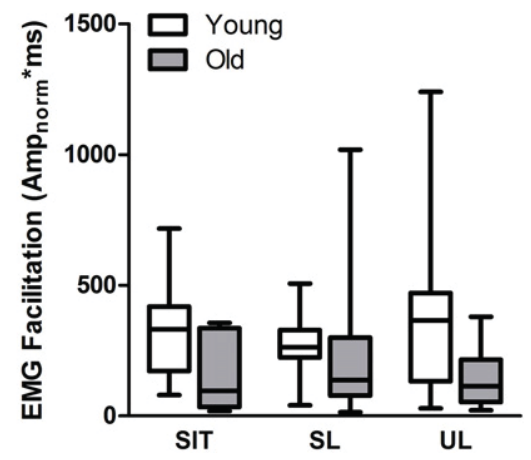

Fig. 3. Group data for young and old adults of (A) soleus EMG suppression and (B) soleus EMG facilitation. Conditions were sitting (SIT), supported leaning (SL), and unsupported leaning (UL). The horizontal line within the box indicates the median value, the box covers the 25 th-75th percentiles, and the whiskers represent the range. ${ }^{*}$ denotes a significant difference ( $\mathrm{p}<0.05$ ). 


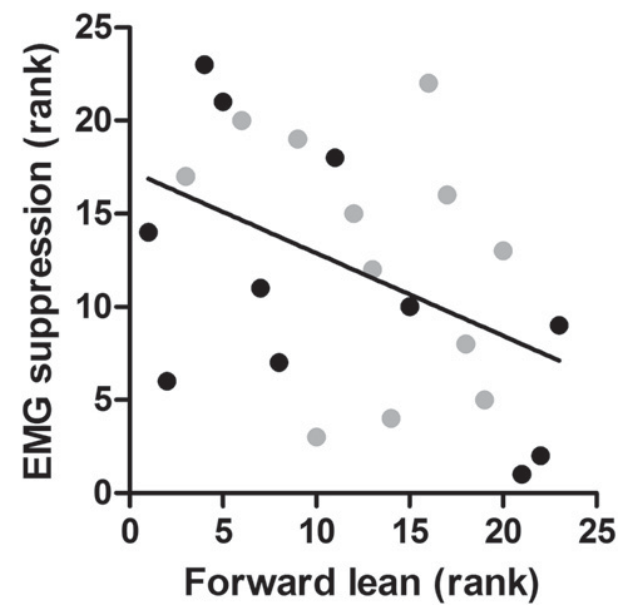

Fig. 4. Correlation between the forward lean and the EMG suppression during unsupported leaning. The forward lean was defined as the CoP position in anteroposterior direction during the unsupported leaning condition as a percentage of the CoP position during a maximum forward lean. The EMG suppression was expressed as a percentage of the EMG suppression during supported leaning. Because of non-normal distribution, the ranks are shown instead of the original data. The regression line illustrates that leaning more forward was correlated with less EMG suppression ( $\mathrm{rho}=-0.44, \mathrm{p}=0.034$ ). Gray and black symbols, respectively, denote young and old adults.

between index finger and thumb muscle activation when healthy adults used these muscles synergistically, mediated at least in part by a decrease of SICI and an increase of recurrent excitation (KouchtirDevanne et al., 2012).

With respect to postural control, previous studies have compared contractions during sitting vs. standing to investigate the influence of contraction aim on corticospinal measures (Soto et al., 2006; Obata et al., 2014; Baudry et al., 2015; Ackermann et al., 1991; Lavoie et al., 1995). However, this method has the limitation that not only contraction aim, but also posture and postural challenge are altered. To the best of our knowledge, the present study is the first to investigate the different factors (contraction aim, postural challenge, and posture) separately. The pattern of modulation in EMG suppression (less suppression during UL compared with SL and SIT) suggests that postural challenge was the most critical factor modulating motor cortical activity, whereas contraction aim and posture had no or only a small influence.

The modulation in EMG suppression between postural challenging and non-challenging contexts observed in the current study is consistent with the literature reporting a decrease in intracortical inhibition and an increase in cortical excitability with an increase in postural task difficulty (Tokuno et al., 2009; Obata et al., 2014; Papegaaij et al., 2014b; Baudry et al., 2015). We found that the modulation was not correlated with motor performance, but it was correlated with the CoP position in relation to the maximum forward lean. This is somewhat surprising, and suggests that it is not merely task difficulty that influences the intracortical circuits, as task difficulty would also be reflected in CoP variability. It seems that instead the modulation in motor cortical activity was related to the threat of losing balance, which increases when moving the center of mass closer to the boundaries of stability. Interestingly, changes in intracortical inhibition and cortical excitability are not always related to level of muscle contraction and are present even when the investigated muscle is relaxed (Obata et al., 2014) or, as in the present study, when muscle activity is similar between conditions (Tokuno et al., 2009). Therefore, we speculate that these modulations are a reflection of an increased readiness state of the central nervous system to counteract possible oncoming perturbations. In behavioral research investigating the effect of expectation and context on postural responses to surface translations or rotations this readiness state is referred to as "central set" (Horak et al., 1989). Whether the modulation in intracortical circuits indeed underlies central set changes for postural responses would be an interesting topic for future research.

\subsection{No age-related differences in modulation of EMG suppression}

To the best of our knowledge, this is the first study using subthreshold TMS in old adults. We found no age-related differences in the modulation of EMG suppression between conditions. These results are consistent with a previous study where we used paired pulse TMS and found similar modulation of SICI between supported and unsupported standing in young and old adults (Papegaaij et al., submitted for publication). However, we did find an age by condition interaction in SICI when comparing standing on foam vs. standing on a rigid platform (Papegaaij et al., 2014b). Differences in postural challenge can explain these different outcomes. When young and old adults perform the same difficult task (standing on foam), old adults will be more unstable likely due to age-related changes in sensory input (Goble et al., 2009; Shaffer and Harrison, 2007), muscle strength (Granacher et al., 2013; Cattagni et al., 2014; Hasson et al., 2014), and nerve conduction velocity (Nardone et al., 1995). The greater relative postural challenge might underlie the greater modulation in intracortical inhibition. In the present study we adjusted postural challenge to personal skill level, by setting the target relative to the maximum. Despite the fact that individual maxima for forward leaning were much lower in old adults, modulation of EMG suppression was similar between young and old adults. This suggests that age does not affect the motor control strategy of reducing intracortical inhibition when posture is challenged. However, the absolute threshold when modulation of intracortical inhibition is required seems to be lower.

\subsection{EMG facilitation}

In most subjects EMG activity became facilitated after the suppression. Similar facilitation has been reported in previous studies but the neural correlates remain unclear (Davey et al., 1994; Petersen et al., 2001, 2011; Seifert and Petersen, 2010). Consistent with Seifert and Petersen (2010), we did not find a relationship between the magnitude of suppression and facilitation in young subjects. However, in old adults greater facilitation was correlated with greater suppression. Therefore, it seems likely that at least part of the facilitation was due to a 'rebound' effect, a synchronous discharge of the motor units after a period of inhibition (Petersen et al., 2011; Turker and Powers, 1999). The facilitation may also be due to activation of intracortical excitatory circuits (Seifert and Petersen, 2010; Petersen et al., 2011).

\subsection{Limitations and future recommendations}

Although subjects received feedback in all conditions, the type of feedback differed between UL (CoP position) and SIT and SL (rectified EMG signal). As the type of feedback can influence subTMS induced EMG suppression (Lauber et al., 2012), we cannot rule out the possibility that the type of feedback played a role in the reduction of EMG suppression during UL. However, there are two reasons why we believe this is not the case. First, Lauber et al. (2012) reported greater EMG suppression during a position-controlled task when compared with a forcecontrolled task. Therefore, greater instead of lower EMG suppression would be expected during UL if the modulation was due to the type of feedback. Second, the correlation between the forward lean and EMG suppression during UL cannot be explained by differences in feedback.

Another limitation is that, although the leaning target was set to each subject's maximum, old compared with young adults still exhibited higher bEMG as a percentage of MVC ( 21 vs $12 \%$ MVC). This was caused by lower MVC values in old adults, as the absolute bEMG was similar in the two age groups. Although contraction at 10 to $40 \%$ of MVC did not affect TMS-induced EMG suppression (Seifert and Petersen, 2010), we nonetheless compared EMG suppression using an 
analysis of covariance with bEMG as a covariate. The condition and age by condition interaction effects remained similar to the initial analyses. Therefore, we conclude that the age-related difference in bEMG did not affect our main results. However, the interpretation of the age main effects with respect to EMG suppression and facilitation requires caution. Future research will clarify whether the age-related differences in EMG suppression and facilitation are still present when examined during muscle contractions of similar intensity, and whether under such conditions the age-related differences in inhibition and facilitation are related to balance performance.

\subsection{Conclusion}

TMS-induced EMG suppression, most likely reflecting intracortical inhibition, was lower during muscle contractions to keep a forward leaning posture than during voluntary contractions while sitting and during supported leaning. This decrease was due to differences in postural challenge, and not due to differences in contraction aim (postural vs. non-postural) or posture (leaning vs. sitting). Even though in absolute terms forward lean was farther in young than in old adults, modulation of TMS-induced EMG suppression was similar between young and old adults. This suggests that age does not affect the motor control strategy of modulating motor cortical activity with increasing postural challenge, but the modulation appears at a lower task difficulty with increasing age.

\section{Acknowledgments}

This work was supported in part by start-up funds from the University Medical Center Groningen, grant number 653013. The authors would like to thank J.B. Waanders and N.E. Lourens for their help with subject recruitment and data collection, E. Smid for his technical assistance, and J. Terpstra for his help with the graphics.

\section{References}

Ackermann, H., Scholz, E., Koehler, W., Dichgans, J., 1991. Influence of posture and voluntary background contraction upon compound muscle action potentials from anterior tibial and soleus muscle following transcranial magnetic stimulation. Electroencephalogr. Clin. Neurophysiol. 81, 71-80.

Baudry, S., Collignon, S., Duchateau, J., 2015. Influence of age and posture on spinal and corticospinal excitability. Exp. Gerontol. 69, 62-69 (doi: S0531-5565(15)00183-7).

Baudry, S., Penzer, F., Duchateau, J., 2014. Input-output characteristics of soleus homonymous ia afferents and corticospinal pathways during upright standing differ between young and elderly adults. Acta Physiol (Oxf.) 210, 667-677. http://dx.doi.org/10. 1111/apha.12233.

Borg, G., 1970. Perceived exertion as an indicator of somatic stress. Scand. J. Rehabil. Med. 2, 92-98.

Cattagni, T., Scaglioni, G., Laroche, D., Van Hoecke, J., Gremeaux, V., Martin, A., 2014. Ankle muscle strength discriminates fallers from non-fallers. Front. Aging Neurosci. 6, 336. http://dx.doi.org/10.3389/fnagi.2014.00336.

Classen, J., Benecke, R., 1995. Inhibitory phenomena in individual motor units induced by transcranial magnetic stimulation. Electroencephalogr. Clin. Neurophysiol. 97, 264-274 (doi: 001346949500099K)

Davey, N.J., Romaiguere, P., Maskill, D.W., Ellaway, P.H., 1994. Suppression of voluntary motor activity revealed using transcranial magnetic stimulation of the motor cortex in man. J. Physiol. 477 (Pt 2), 223-235.

Devanne, H., Cohen, L.G., Kouchtir-Devanne, N., Capaday, C., 2002. Integrated motor cortical control of task-related muscles during pointing in humans. J. Neurophysiol. $87,3006-3017$

Di Lazzaro, V., Restuccia, D., Oliviero, A., Profice, P., Ferrara, L., Insola, A., Mazzone, P., Tonali, P., Rothwell, J.C., 1998. Magnetic transcranial stimulation at intensities below active motor threshold activates intracortical inhibitory circuits. Exp. Brain Res. 119, 265-268.

Dominici, F., Popa, T., Ginanneschi, F., Mazzocchio, R., Rossi, A., 2005. Corticomotoneuronal output to intrinsic hand muscles is differentially influenced by static changes in shoulder positions. Exp. Brain Res. 164, 500-504. http://dx.doi.org/10. 1007/s00221-005-2270-5.

Ginanneschi, F., Del Santo, F., Dominici, F., Gelli, F., Mazzocchio, R., Rossi, A., 2005. Changes in corticomotor excitability of hand muscles in relation to static shoulder positions. Exp. Brain Res. 161, 374-382. http://dx.doi.org/10.1007/s00221-004-2084-x.

Goble, D.J., Coxon, J.P., Wenderoth, N., Van Impe, A., Swinnen, S.P., 2009. Proprioceptive sensibility in the elderly: degeneration, functional consequences and plasticadaptive processes. Neurosci. Biobehav. Rev. 33, 271-278. http://dx.doi.org/10. 1016/j.neubiorev.2008.08.012
Granacher, U., Gollhofer, A., Hortobagyi, T., Kressig, R.W., Muehlbauer, T., 2013. The importance of trunk muscle strength for balance, functional performance, and fall prevention in seniors: a systematic review. Sports Med. 43, 627-641. http://dx.doi. org/10.1007/s40279-013-0041-1.

Guralnik, J.M., Simonsick, E.M., Ferrucci, L., Glynn, R.J., Berkman, L.F., Blazer, D.G., Scherr, P.A., Wallace, R.B., 1994. A short physical performance battery assessing lower extremity function: association with self-reported disability and prediction of mortality and nursing home admission. J. Gerontol. 49, M85-M94.

Hasson, C.J., van Emmerik, R.E., Caldwell, G.E., 2014. Balance decrements are associated with age-related muscle property changes. J. Appl. Biomech. 30, 555-562. http://dx. doi.org/10.1123/jab.2013-0294.

Hebbal, G.V., Mysorekar, V.R., 2006. Evaluation of some tasks used for specifying handedness and footedness. Percept. Mot. Skills 102, 163-164.

Heuninckx, S., Wenderoth, N., Swinnen, S.P., 2008. Systems neuroplasticity in the aging brain: recruiting additional neural resources for successful motor performance in elderly persons. J. Neurosci. 28, 91-99. http://dx.doi.org/10.1523/JNEUROSCI.330007.2008 .

Horak, F.B., Diener, H.C., Nashner, L.M., 1989. Influence of central set on human postural responses. J. Neurophysiol. 62, 841-853.

Howatson, G., Taylor, M.B., Rider, P., Motawar, B.R., McNally, M.P., Solnik, S., DeVita, P., Hortobagyi, T., 2011. Ipsilateral motor cortical responses to TMS during lengthening and shortening of the contralateral wrist flexors. Eur. J. Neurosci. 33, 978-990. http://dx.doi.org/10.1111/j.1460-9568.2010.07567.x.

Kouchtir-Devanne, N., Capaday, C., Cassim, F., Derambure, P., Devanne, H., 2012. Taskdependent changes of motor cortical network excitability during precision grip compared to isolated finger contraction. J. Neurophysiol. 107, 1522-1529. http://dx.doi. org/10.1152/jn.00786.2011.

Lauber, B., Leukel, C., Gollhofer, A., Taube, W., 2012. Time to task failure and motor cortical activity depend on the type of feedback in visuomotor tasks. PLoS One 7, e32433. http://dx.doi.org/10.1371/journal.pone.0032433.

Lavoie, B.A., Cody, F.W., Capaday, C., 1995. Cortical control of human soleus muscle during volitional and postural activities studied using focal magnetic stimulation. Exp. Brain Res. 103, 97-107.

Magnus, R., 1926. Some results of studies in the physiology of posture. Lancet 2, 585-588.

Malouin, F., Richards, C.L., Jackson, P.L., Dumas, F., Doyon, J., 2003. Brain activations during motor imagery of locomotor-related tasks: a PET study. Hum. Brain Mapp. 19, 47-62.

Mattay, V.S., Fera, F., Tessitore, A., Hariri, A.R., Das, S., Callicott, J.H., Weinberger, D.R., 2002. Neurophysiological correlates of age-related changes in human motor function. Neurology 58, 630-635.

Nardone, A., Siliotto, R., Grasso, M., Schieppati, M., 1995. Influence of aging on leg muscle reflex responses to stance perturbation. Arch. Phys. Med. Rehabil. 76, 158-165.

Obata, H., Sekiguchi, H., Ohtsuki, T., Nakazawa, K., 2014. Posture-related modulation of cortical excitability in the tibialis anterior muscle in humans. Brain Res. 1577, 29-35. http://dx.doi.org/10.1016/j.brainres.2014.06.027.

Ortu, E., Deriu, F., Suppa, A., Tolu, E., Rothwell, J.C., 2008. Effects of volitional contraction on intracortical inhibition and facilitation in the human motor cortex. J. Physiol. 586, 5147-5159. http://dx.doi.org/10.1113/jphysiol.2008.158956.

Papegaaij, S., Taube, W., Baudry, S., Otten, E., Hortobagyi, T., 2014a. Aging causes a reorganization of cortical and spinal control of posture. Front. Aging Neurosci. 6, 28. http:// dx.doi.org/10.3389/fnagi.2014.00028.

Papegaaij, S., Taube, W., Hogenhout, M., Baudry, S., Hortobagyi, T., 2014b. Age-related decrease in motor cortical inhibition during standing under different sensory conditions. Front. Aging Neurosci. 6, 126. http://dx.doi.org/10.3389/fnagi.2014.00126.

Papegaaij, S., Baudry, S., Négyesi, J., Taube, W., Hortobágyi, T., 2015. Intracortical inhibition in the soleus muscle is reduced during the control of upright standing in both young and old adults. (submitted for publication).

Petersen, N.T., Butler, J.E., Marchand-Pauvert, V., Fisher, R., Ledebt, A., Pyndt, H.S., Hansen, N.L., Nielsen, J.B., 2001. Suppression of EMG activity by transcranial magnetic stimulation in human subjects during walking. J. Physiol. 537, 651-656.

Petersen, N.C., Taylor, J.L., Murray, N.P., Gandevia, S.C., Butler, J.E., 2011. Differential effects of low-intensity motor cortical stimulation on the inspiratory activity in scalene muscles during voluntary and involuntary breathing. Respir. Physiol. Neurobiol. 175, 265-271. http://dx.doi.org/10.1016/j.resp.2010.11.014.

Rantalainen, T. Weier, A. Leung M., Brandner, C., Spittle, M., Kidgell, D. 2013. Shortinterval intracortical inhibition is not affected by varying visual feedback in an isometric task in biceps brachii muscle. Front. Hum. Neurosci. 7, 68. http://dx.doi.org/ 10.3389/fnhum.2013.00068.

Reis, J., Swayne, O.B., Vandermeeren, Y., Camus, M., Dimyan, M.A., Harris-Love, M., Perez, M.A., Ragert, P., Rothwell, J.C., Cohen, L.G., 2008. Contribution of transcranial magnetic stimulation to the understanding of cortical mechanisms involved in motor control. J. Physiol. 586, 325-351 (doi: jphysiol.2007.144824).

Ridding, M.C., Taylor, J.L., Rothwell, J.C., 1995. The effect of voluntary contraction on cortico-cortical inhibition in human motor cortex J. Physiol 487 (Pt 2), 541-548.

Schneider, C. Devanne, H., Lavoie, B.A., Capaday, C., 2002. Neural mechanisms involved in the functional linking of motor cortical points. Exp. Brain Res. 146, 86-94. http://dx. doi.org/10.1007/s00221-002-1137-2.

Seifert, T., Petersen, N.C., 2010. Changes in presumed motor cortical activity during fatiguing muscle contraction in humans. Acta Physiol (Oxf.) 199, 317-326. http://dx.doi. org $/ 10.1111 / \mathrm{j} .1748-1716.2010 .02098 . x$

Shaffer, S.W., Harrison, A.L., 2007. Aging of the somatosensory system: a translational perspective. Phys. Ther. 87, 193-207. http://dx.doi.org/10.2522/ptj.20060083.

Sharples, S.A., Kalmar, J.M., 2012. Modulation of cortical excitability and interhemispheric inhibition prior to rhythmic unimanual contractions. J. Neurosci. Methods 210 178-186. http://dx.doi.org/10.1016/j.jneumeth.2012.07.018.

Sherrington, C.S., 1910. Flexion-reflex of the limb, crossed extension-reflex, and reflex stepping and standing. J. Physiol. 40, 28-121. 
Sidhu, S.K., Cresswell, A.G., Carroll, T.J., 2013. Short-interval intracortical inhibition in knee extensors during locomotor cycling. Acta Physiol (Oxf.) 207, 194-201. http://dx.doi. org/10.1111/apha.12004.

Soto, O., Valls-Sole, J., Shanahan, P., Rothwell, J., 2006. Reduction of intracortical inhibition in soleus muscle during postural activity. J. Neurophysiol. 96, 1711-1717. http://dx. doi.org/10.1152/jn.00133.2006.

Taube, W., Schubert, M., Gruber, M., Beck, S., Faist, M., Gollhofer, A., 2006. Direct corticospinal pathways contribute to neuromuscular control of perturbed stance. J. Appl. Physiol. 101, 420-429. http://dx.doi.org/10.1152/japplphysiol.01447.2005.
Tokuno, C.D., Taube, W., Cresswell, A.G., 2009. An enhanced level of motor cortical excitability during the control of human standing. Acta Physiol (Oxf.) 195, 385-395. http://dx.doi.org/10.1111/j.1748-1716.2008.01898.x.

Turker, K.S., Powers, R.K., 1999. Effects of large excitatory and inhibitory inputs on motoneuron discharge rate and probability. J. Neurophysiol. 82, 829-840.

Zuur, A.T., Lundbye-Jensen, J., Leukel, C., Taube, W., Grey, M.J., Gollhofer, A., Nielsen, J.B., Gruber, M., 2010. Contribution of afferent feedback and descending drive to human hopping. J. Physiol. 588, 799-807. http://dx.doi.org/10.1113/jphysiol. 2009.182709. 